Review

\title{
Ketogenic Therapy for Major Depressive Disorder: A Review of Neurobiological Evidence
}

Dana Shamshtein ${ }^{1}$, Timur Liwinski ${ }^{2, *}$

1. School of Psychological Sciences, Tel Aviv University, Ramat Aviv, POB 39040, 69978, Tel Aviv, Israel; E-Mail: dana.sham@gmail.com

2. Immunology Department, Weizmann Institute of Science, Rehovot, 7610001, Israel; E-Mails: liwinskt@gmail.com; timur.liwinski@weizmann.ac.il

* Correspondence: Timur Liwinski; E-Mails: liwinskt@gmail.com; timur.liwinski@weizmann.ac.il

Academic Editor: Jennifer Keogh

Special Issue: Lifestyle Modification in Pathophysiology

Recent Progress in Nutrition

2022, volume 2 , issue 1

doi:10.21926/rpn.2201003
Received: December 06, 2021

Accepted: February 07, 2022

Published: February 17, 2022

\begin{abstract}
Major depressive disorder (MDD) is a debilitating illness that places a great burden on global health. A ketogenic diet, which has traditionally been used for treating refractory epileptic disorders, is being increasingly explored for its potential of alleviating other neuropsychiatric conditions. Providing an up-to-date summary on the ketogenic diet's potential to alleviate MDD, its neurobiological basis, and preliminary clinical evidence. A structured literature survey was performed, and a narrative review was written. Basic science provides cues for the biological mechanisms underlying the antidepressant effects of ketosis. Ketosis may circumvent frontal cortical glucose hypometabolism, reduce oxidative stress and neuroinflammation, and restore neurotransmitter homeostasis by increasing gammaaminobutyric acid (GABA) levels and decreasing excitatory neurotransmitter levels. Another interesting domain affected by ketosis and potentially linked to mood and mental health is the gut microbiome. Emerging clinical evidence supports the efficacy of ketosis induction in treating patients with mood disorders. Evidence supports the antidepressant potential of the
\end{abstract}


ketogenic diet. However, further substantial research into the underlying mechanisms of action and clinical effects is required.

\section{Keywords}

Diet; nutrition; nutraceuticals; mental health; depression major depressive disorder; ketogenic diet

\section{Introduction}

Depressive disorders are among the leading causes of years lived with disability (YLDs) among women and men of all age groups worldwide [1]. The persistence of depressive disorders as a leading cause of health loss over the past three decades is particularly concerning, considering its association with self-harming tendencies [1]. Major depressive disorder (MDD) is a debilitating illness characterized by at least one discrete depressive episode with low mood, decreased interests, impaired cognitive function, and vegetative symptoms, such as disturbed sleep or appetite changes, lasting a minimum of two weeks [2]. In addition, MDD is associated with an increased risk of poor health outcomes in other domains, such as cardiovascular disorders []. The etiology of MDD is multifactorial, comprising both genetic and environmental factors, such as the experience of physical or emotional abuse during childhood [2]. Psychotherapy and psychopharmacological treatments are effective in treating MDD. However, despite repeated treatment attempts, MDD persists in approximately $30 \%$ of patients [4, 5]. Moreover, antidepressant pharmacological treatments may cause significant side effects [5], which could be potentially circumvented by alternative approaches. Considering the high personal and societal burden of MDD, there is a strong incentive in identifying alternative or augmentative treatments.

In Western societies, the prevalence of mental disorders has increased in parallel with the nutritional deterioration associated with typical Western diets [6]. Foods rich in valuable macronutrients and microelements are being increasingly replaced by ultra-processed foods, which are associated with increased health risks, including type-2 diabetes, cardiovascular diseases, cancers, and depression [7]. There has been a longstanding interest in dietary and nutraceutical interventions for alleviating mental disorders [8]. Several interventional studies have attempted to alleviate depressive symptoms by utilizing nutrients, mainly focusing on omega-3 fatty acids, folate, vitamin B12, magnesium, zinc, iron, and amino acids such as 5-hydroxytryptophan and L-tryptophan [9-16].

In modern medicine, ketogenic diets have been used for almost a century to treat refractory epilepsy [17]. The use of a ketogenic diet for therapeutic means in modern medicine dates back to the 1920s [18]. A ketogenic diet is high in fat and low in carbohydrates and aims to achieve ketosis [16]. Ketosis is a physiological condition characterized by increased levels of circulating ketone bodies achieved by fasting or a significantly reduced net carbohydrate intake [18]. Three to four days of no carbohydrate consumption cause the brain to switch to an alternative energy source. In this condition, the tissues in the body and brain utilize ketone bodies rather than glucose as the primary fuel for energy generation [19]. Ketone bodies are mainly produced in a process termed "ketogenesis", which occurs in the mitochondrial matrix of hepatocytes [20]. The normal 
concentration of ketone bodies in carbohydrate-consuming individuals is $<0.03 \mathrm{mmol} / \mathrm{L}$. The brain begins utilizing ketone bodies as fuel once a concentration of approximately $4 \mathrm{mmL} / \mathrm{L}$ of ketone bodies is reached in the systemic circulation [17]. The ketone bodies that are consumed by the tissues in the body and brain as the alternative fuel include acetoacetate, $\beta$-hydroxybutyric acid, and acetone. Different practical approaches and implementations of the ketogenic diet are recommended, such as those reported by Carroll and Koenigsberger [21]. Ketogenic diet is now being increasingly explored for treating other neuropsychiatric conditions, including mental disorders [22].

The present study aimed to explore the neurobiological evidence in support of improvement in depression upon the adoption of a ketogenic diet and the underlying biological mechanisms uncovered through in vitro and in vivo studies. Moreover, a bench-bedside perspective summarizing the available preliminary clinical evidence is provided.

\section{Methods}

\subsection{Data Sources and Search Strategy}

The databases PubMed, EMBASE, Science Citation Index, Cochrane Library, PsycINFO, and clinicaltrials.gov were searched from inception ( $1^{\text {st }}$ August 2021 ) to $17^{\text {th }}$ January 2022 , using the following terms: "depression", "depressed", "major depressive disorder", "mood disorders", "psychiatry", "ketogenic diet", "ketosis", "ketogenesis", "ketone bodies", "ketonemia”, "low carbohydrate", "high fat", "fasting", "acetoacetic acid", "acetone", "beta-hydroxybutyric acid", "acetyl-CoA", "oxidative stress", "microbiome", "microbiota", "inflammation", "glucose metabolism", "glucose hypometabolism", "GABA", "glutamate", "mitochondria", "mitochondrial dysfunction", "animal", "mouse", "mice", "rodent", "clinical trial", "randomized controlled trial", and "observational study". The afore-stated search terms were concatenated using different combinations of Boolean operators.

\subsection{Selection Criteria}

Two investigators (D.S. and T.L.) independently examined the titles and abstracts for relevance to the objective of the present study. The inclusion decision was established based on discussion and consensus. After identifying relevant tags, the abstracts of these articles were examined to decide if the study contained suitable and eligible material. A manual cross-reference search of bibliographies was also conducted to screen for additional potentially relevant articles. Only fulltext articles were selected. The survey was confined to the articles published in English.

\subsection{Quality Assessment}

Original literature was scrutinized according to specific quality criteria. Cohort and case-control studies were evaluated using the Newcastle-Ottawa scale [23]. Research articles reporting in vivo experimentation in animal studies were assessed according to the ARRIVE guidelines [24]. 


\section{Neurobiological Basis of Ketogenic Diet's Efficacy}

\subsection{Impaired Cerebral Glucose Metabolism}

The ketogenic diet circumvents deficiencies in the cellular energy metabolism resulting from reduced glucose uptake and inefficient glycolysis by inducing a steep rise in the levels of circulating ketone bodies and replacing glucose as the dominant fuel source altogether [25]. This way, ketone bodies might restore mitochondrial energy metabolism and homeostasis. Glucose is usually the brain's primary source of energy. However, accumulating evidence demonstrates that individuals with depression exhibit marked alterations in cerebral glucose metabolism. Such findings are generally based on functional neuroimaging with [18F]-fluoro-deoxyglucose positron emission tomography scans.

Martinot et al. have reported significant left-right prefrontal asymmetry in the patients before but not after successful tricyclic antidepressant treatment. Furthermore, patients in a depressed state reportedly exhibit significant hypofrontality and whole-cortex hypometabolism, which persisted after treatment commencement, despite clinical improvement [25].

Dunn et al. correlated the symptom clusters in Beck's depression inventory to glucose metabolism in various brain regions. In both unipolar and bipolar depression, the psychomotoranhedonia cluster was correlated with lower glucose metabolism in the right insula, claustrum, anteroventral caudate/putamen, and temporal cortex. Notably, in patients with unipolar depression, the negative cognitions cluster was correlated with lower absolute glucose metabolism in the frontal brain, specifically bilaterally in the frontal poles and right dorsolateral frontal cortex, as well as in the supracallosal cingulate [26].

Kimbrell and colleagues reported similar conclusions when they studied the associations of [18F]fluoro-deoxyglucose metabolization with Hamilton's depression scores. In that study, regions of the frontal, cingulate, insula, and temporal cortex were hypometabolic in severely depressed individuals with treatment-resistant unipolar depression [27].

Similar results were reported by Fujimoto et al. reported in patients with late-onset depression, who exhibited decreased relative glucose metabolism in the prefrontal, cingulate, and parietal regions of both hemispheres and the right temporal region, along with increased relative metabolism in the occipital pole, vermis, cerebellum, dorsal-frontal, and central convexity regions and basal ganglia in both hemispheres, in depressed patients, compared to healthy controls [28].

The insulin-independent glucose transporter 1 (GLUT1) plays a critical role in the glucose metabolism occurring in the human brain. Kahl et al. employed bisulfite sequencing to study the methylation patterns of glucose transporters in depressed individuals. The authors observed a significantly increased GLUT1 methylation (and thus inhibition of expression) in the inpatients with depression compared to healthy controls. After inpatient treatment, GLUT1 methylation was significantly lowered in the treatment responders compared to the levels in non-responders [29]. These results highlight that cerebral glucose uptake from blood circulation might be compromised in patients with MDD, suggesting that switching to alternative primary sources of energy, such as ketone bodies, could potentially alleviate cerebral dysmetabolism in depressed individuals. 


\subsection{Impact on GABA and Glutamate Balance}

Depression is the most common comorbidity in epilepsy, affecting up to $62 \%$ of patients [30]. The manifestation of depression in epilepsy is a complex process involving several underlying neurochemical and psychosocial determinants. Glutamatergic and GABAergic dysfunction in epileptic disorders has been recognized for a long time now [30]. Although the "monoamine theory" implicating altered serotonin and norepinephrine in depression has dramatically advanced the understanding and treatment of mood disorders, it does not present a complete picture of neurotransmitter alterations in depressed individuals [31]. In vivo magnetic spectroscopy studies in depressed individuals have revealed altered cortical glutamate levels, prompting the hypothesis of excessive glutamate-induced excitation in depression [31]. Consistent with this hypothesis, several anti-glutamatergic agents, such as riluzole and lamotrigine, have demonstrated antidepressant potency [31]. GABA is an inhibitory neurotransmitter present almost exclusively in the central nervous system. Evidence suggests that reduced GABAergic activity accompanies depression, similar to epilepsy [32]. Moreover, there is evidence that ketosis modulates brain function by altering cerebral glutamate metabolism [33]. In ketosis, the astrocyte metabolism is enhanced, which results in an increased conversion of glutamate into glutamine [34]. This phenomenon may increase glutamate removal and reduce glutamate-induced excitability, which could lead to a further efficient glutamate exchange to GABA, increasing GABAergic activity [35]. This neurochemical model might explain the efficacy of ketosis in treating epilepsy and, considering its shared pathophysiological features with depression, provides a firm explanation of how ketosis improves depressive symptoms. The understanding of the mechanism through which ketosis improves epilepsy and mood would pave the way for future clinical trials.

\subsection{Mitochondrial Dysfunction and Oxidative Stress}

Oxidative stress is defined as an imbalance between the production of reactive oxygen species and antioxidant defenses [36]. Mitochondrial dysfunction associated with oxidative stress may cause abnormal brain function, which may lead to depression [37]. Mitochondria, the principal locations for energy metabolism in cells, are responsible for producing and eliminating reactive oxygen species, processes that are significant in the pathogenesis of neurodegenerative diseases [38]. Ketosis impacts mitochondria and oxidative processes through various mechanisms. Ketone bodies regulate mitochondrial functions and redox signaling through the induction of low redox signaling molecules, including $\mathrm{H}_{2} \mathrm{O}_{2}$ and electrophiles such as 4-hydroxynonenal (4-HNE), which trigger adaptive-pathway molecules, such as the protective transcription factor NF E2-related factor 2 (Nrf2). This process may ultimately increase the levels of antioxidants (e.g., GSH) and detoxification enzymes, thereby improving brain function and alleviating neurodegeneration [39]. An interesting hypothesis is that the alleviation of mitochondrial dysfunction and oxidative stress could be among the mechanisms through which ketosis reduces epileptic seizures [40]. Together, these data provide a strong argument in favor of the efficacy of ketosis against depression.

\subsection{Ketosis and Inflammation}

While depression is probably not a purely autoinflammatory condition, there is ample evidence in support of the association between depression and inflammation [41]. This observation has been 
replicated several times and reviewed extensively [42]. For instance, the severity of depression correlates with the levels of pro-inflammatory serum cytokines IL-1 $\beta$, TNF- $\alpha$, and IL-8 [43]. In a lipopolysaccharide-induced fever model in rats, animals fed with a ketogenic diet exhibited lower pro-inflammatory cytokine levels compared to controls, which was probably mediated by an increase in anti-inflammatory n-3 PUFA derivatives [44]. Similarly, mice under a systemic lipopolysaccharide exposure challenge exhibited lower levels of circulating IL-6 and TNF- $\alpha$ [45]. A murine model of neurotoxicity induced by 1-methyl-4-phenyl-1,2,3,6-tetrahydropyridine (MPTP) exhibited lower levels of pro-inflammatory cytokines interleukin-1 $\beta$, interleukin-6, and tumor necrosis factor-alpha in the brain under a ketogenic feeding regime [46].

Moreover, the ketone body $\beta$-hydroxybutyrate was demonstrated to alleviate symptoms of depression in rodents together with a reduction in the levels of neuroinflammatory markers [47, 48]. A single dose of $\beta$-hydroxybutyrate administration reduced the levels of hippocampal IL-1 $\beta$ and TNF- $\alpha$, potentially through the inhibition of the NLRP3 inflammasome [48]. Microglia are brainspecific immune cells implicated in the regulation of brain development, synaptic plasticity, and mood [49]. Microglial alterations are proposed to play a critical pathophysiological role in depression [49]. Huang et al. have demonstrated that in mice with lipopolysaccharide-induced depressive behavior, $\beta$-hydroxybutyrate exerted an antidepressant effect by promoting microglial ramification (a morphological change impacting immune function) [47].

Together, these findings provide evidence in support of the antidepressant effects of ketone bodies via their immunomodulatory actions. Further studies are, however, required to elucidate the mechanistic links among ketone bodies, immunity, and mood.

\subsection{Ketosis and Gut Microbiome}

In the past decade, the gut microbiota has become a research "hotspot" in science, with countless studies published on the implication of the gut microbiome in virtually every organ system and disease [50]. The relationship between gut microbial metabolism and mental health is one of the most intriguing and controversial topics in microbiome research. Growing evidence suggests that the configuration of gut microbiota may alter brain physiology and behavior in various brain disorders, including depression [51]. A causal role of the gut microbiota in depression is supported by the findings in animal model-based studies [52]. The mechanisms underlying this connection remain uncertain, though, and are likely to be of high complexity. Several hypotheses have been proposed, including the bidirectional interaction of microbial metabolites released into the host circulation with the hypothalamus-pituitary-adrenaline (HPA) axis and the immune system, the signaling of intestinal microbial metabolites to the enteric nervous system, and the modulation of behavior via vagal afferents [53].

In an extensive microbiome population survey (Flemish Gut Flora Project, $n=1,054$ ), which included validation in an independent dataset $(n=1,070)$, certain potentially beneficial bacteria, such as those belonging to Faecalibacterium and Coprococcus, were consistently associated with the indicators of a higher quality of life. Together with Dialister, Coprococcus spp. were also depleted in depression, even after adjustment for the confounding effects of antidepressants [54]. It appears that microbiome alterations observed in the patients with depression largely overlap with the alterations typically detected in other chronic diseases, such as metabolic syndrome. Such alterations include the decline of certain bacterial genera, such as Bacteroides, Coprococcus, Dorea, 
Ruminococcus, and Faecalibacterium, and the bloom of others such as those belonging to the Fusobacteria and Proteobacteria phyla [53].

Diet is one of the significant determinants of microbiome configuration. For instance, a switch from a plant-based diet to a diet composed mainly of animal products could alter the microbiome composition tremendously within a short duration [55]. The alteration of the gut microbiome after a ketogenic diet is well established in animal models and various patient populations with brain disorders, including children with refractory epilepsy, patients with Alzheimer's disease, and patients with multiple sclerosis [56-60]. Olson et al. went a step further and proposed that the gut microbiota mediated the anti-seizure effects of a ketogenic diet [61]. Growing evidence suggests that a ketogenic diet fosters microbial recovery from the commonly observed adverse microbiome features, such as those in metabolic syndrome and depression [62]. Thus, a ketogenic diet may alleviate the burden of depressive symptoms by restoring the balance of gut microbiota.

These data provide a strong rationale for studying the effects of a ketogenic diet on the gut microbiota and symptom improvement in patients with depression and animal models with depression-like behaviors.

\section{Evidence for Mood Modulation using Ketogenic Diet from Animal Studies}

Despite the inherent difficulties and pitfalls in modeling a complex human phenomenon, such as depression, using animals, studies in rodents have provided valuable insights into the potential pathophysiological mechanisms and clues for novel treatments for $\operatorname{MDD}[63,64]$.

In a rat model of depression-like behaviors, proxy immobility could be improved using a ketogenic diet, indicating that ketosis improves "behavioral despair" [65].

In another animal study, feeding ketone salt and ketone salts mixed with medium-chain triglycerides improved anxiety-associated behaviors in Sprague-Dawley and WAG/Rij rats [66]. This feeding regimen achieved ketosis within seven days. Kovacs et al. demonstrated that the effects of a ketogenic diet could be neutralized by administering the adenosine A1 receptor antagonist, 1,3dipropyl-8-cyclopentylxanthine, which indicated a contribution of adenosine A1 receptors in the effect of ketogenic diet treatment [67].

In an intriguing study on mice, Sussman et al. revealed that in utero exposure to the gestational ketogenic diet of the mother mouse modulated the offspring's brain structures and protects against anxious and depression-associated behaviors later in adulthood, even though the offspring were fed a standard chow after birth [68]. Growing evidence from murine studies suggests that ketosis has a complex and lasting brain modulatory impact on functions such as hippocampal memory [69].

In a recent animal study on Balb/c mice, Cox and colleagues demonstrated that a ketogenic diet combined with regular exercise decreased anxiety and depressive behaviors. The reduction in depression burden was correlated with $\beta$-hydroxybutyrate levels, which supported the causal role of ketone bodies in behavioral changes. Interestingly, reduction in depression was also correlated with the LDL/HDL cholesterol ratio, insulin, and glucose, thereby linking mood improvement to favorable metabolic changes [70].

Notably, the anti-inflammatory and antidepressant effects demonstrated in mice and rats upon the application of $\beta$-hydroxybutyrate (refer to section 'ketosis and inflammation') $[47,48]$ highlight that inducing dietary ketosis might not even be necessary to achieve therapeutic effects, and rather the administration of single ketone bodies might represent a further feasible treatment option. 
The evidence for the improvement in depressive symptoms and the underlying mechanisms is limited. However, the existing data are encouraging and warrant further mechanistic studies on the beneficial neuromodulator effects of ketosis.

\section{Clinical Evidence}

While the ketogenic diet has been approved for treating refractory epilepsy [71], the emerging evidence indicates a positive impact on psychological burden as well. Although there are currently, to the best of our knowledge, no direct-controlled studies available on the effects of the ketogenic diet on clinical depression, several lines of evidence from human studies indicate a positive impact of this diet type on mood and cognition [72-77].

A randomized controlled study investigated the changes in the behavior and cognition of children and adolescents with refractory epilepsy during the first four months on a ketogenic diet. It was revealed that compared to a regular diet with standard care, the ketogenic diet reduced anxiety and improved both mood and cognition [73]. Specifically, "activated attitude" and "cognitive activation" were observed in both children and adolescents under the ketogenic diet. The study found no adverse clinical effects associated with ketosis [73]. Shegelman et al. studied the psychiatric effects of a ketogenic diet (modified Atkins diet) in adult patients with chronic epilepsy $(n=100)$ and reported that this ketogenic diet could favorably impact the psychological state, with a longer duration on the ketogenic diet being associated with lesser anxiety and depression [77].

McClernon and colleagues compared the ketogenic diet with a low-fat diet in terms of effects on mood, hunger, and self-reported symptoms [75]. Although both diets were effective and caused a notable improvement in a broad range of symptoms, the ketogenic diet exhibited a greater degree of improvement in negative symptoms and hunger. Additionally, the authors disproved that weight loss alone caused mood improvements [75].

It is hypothesized that the ketogenic diet could be utilized as a mood stabilizer in bipolar disorder. A few factors explain this effect. For instance, the changes in the brain energy reported under the ketogenic diet change the global cerebral hypometabolism, a characteristic of the brain of depressed or manic patients. Furthermore, extracellular alternations are believed to reduce intracellular sodium concentrations in ketosis cases, the latter being a common feature of mood stabilizers [72]. In addition, a case study by Phelps et al. demonstrated that a ketogenic diet may act as a mood stabilizer in patients with type II bipolar disorder [74]. Patients who maintained the diet and reached a prolonged state of ketosis achieved mood stabilization superior to that accomplished using medication [74]. Moreover, the patients reported a subjective improvement explicitly attributed to ketosis. The authors attributed this effect to the blood acidification caused by ketosis, which resulted in reduced activity-dependent intracellular sodium levels. This phenomenon also occurs in effective mood-stabilizing pharmacotherapy, further corroborating that ketosis is a potential alternative to pharmacological mood stabilizers [74].

However, not all existing studies confirm the positive effects of a ketogenic diet on mood and psychological well-being. A randomized crossover trial comparing healthy individuals who sustained nutritional ketosis for three weeks to healthy individuals fed with an isocaloric high-carbohydrate low-fat diet reported no difference in mood, cognitive performance, or subjective sleep quality between the two groups of subjects [76]. 
Further clinical research is warranted to explore the effects of the ketogenic diet on mood, cognitive function, and symptoms in MDD. The extensive feasibility and safety data available from human studies for other indications, such as epilepsy, provide a strong rationale for conducting randomized controlled trials investigating ketogenic diet in MDD.

\section{Discussion}

The ketogenic diet has already been approved for pediatric refractory epilepsy and is emerging as an efficient treatment option for other neuropsychiatric disorders as well (reviewed in [78]). The systemic impact of the ketogenic diet on the multiple pathways involved in disease pathogenesis, such as brain metabolism, neurotransmitter balance, inflammation, and gut microbiome, highlights the potential mechanisms of the efficacy of this diet (Figure 1). Overall scarce although encouraging evidence from animal studies merits further in-depth mechanistic research on the effects of ketosis on brain functioning and mood. Nonetheless, the understanding of the mechanisms underlying the effects of ketone bodies on mood and depression-associated physiological dysfunction remains relatively shallow and fragmentary. Therefore, further sophisticated and rigorously controlled study designs exploring animal models are warranted. In this context, exploring the links between the various pathophysiological domains modified by ketosis would be of great interest.

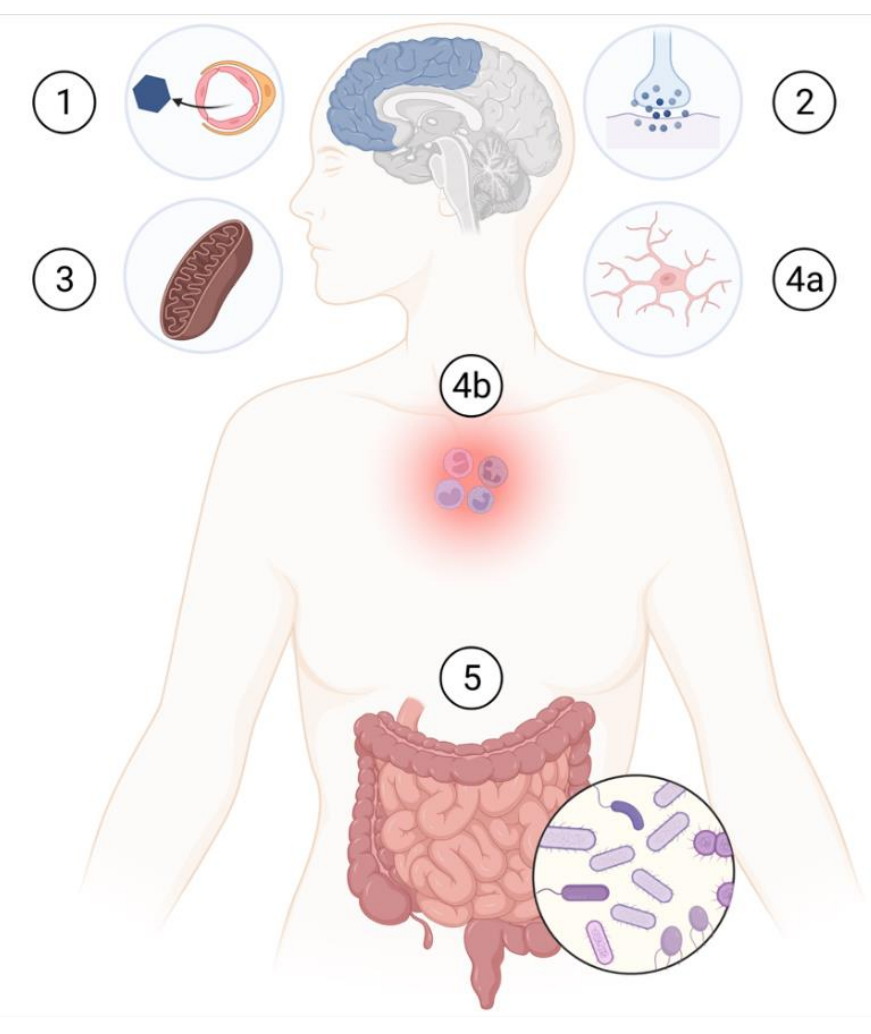

Figure 1 Schematic illustrating and summarizing the major pathomechanisms assumed to play a role in major depressive disorder and alleviated by ketogenic therapy. (1) Frontal glucose hypometabolism, potentially caused by diminished GLUT1 expression; (2) Dysbalanced GABA/glutamate neurotransmitter signaling; (3) Oxidative stress and mitochondrial dysfunction; (4) Inflammation, including (4a) cerebral microglial dysfunction and (4b) low-grade systemic inflammation; (5) Perturbed gut microbiome configuration. 
The ketogenic diet has been proven to be a feasible, safe, and affordable multi-target therapeutic intervention with beneficial pleiotropic systemic effects in humans $[79,80]$. The main shortcoming of the existing literature related to this field is the lack of a direct correlation between the administration of a ketogenic diet and symptom alleviation in depressed patients. However, the data available for the conditions representing typical comorbidities or intersecting pathological features provide a clue for the potentially beneficial effects of the ketogenic diet against MDD. Further research is, however, required to unravel the impact of the ketogenic diet on neurometabolic dysfunction, inflammation, and commensal microbiota alterations associated with depressed human subjects. A deeper understanding of these processes would pave the way for developing strategies that would maximize therapeutic efficacy. Preliminary evidence from the currently reported human clinical studies is too limited to recommend a ketogenic diet as the treatment for MDD. Nevertheless, the available evidence strongly supports the implementation of randomized controlled trials involving the use of the ketogenic diet in depressed populations. In order to advance the clinical research on the ketogenic diet, further precise definitions and a higher grade of standardization have to be established. Currently, there is no consensus on what defines a ketogenic diet in humans, with the lipid-to-non-lipid ratios reported in the literature being highly variable. Moreover, no commonly accepted standard exists for a precise dietary regimen, unpalatable food choices, and the required duration of the dietary course. These shortcomings warrant the establishment of a research task force that would elaborate on a detailed recommendation for the clinical trials involving the use of a ketogenic diet [81]. When designing clinical trials for patients with mental health problems, researchers must pay attention to feasibility and practical implementation issues. Evidence suggests that mentally unwell patients may experience difficulties in measuring food portions and meeting macronutrient targets [82]. Studies on the ketogenic diet in depressed individuals should also assess the importance of covariables that could modify the efficacy of the ketogenic diets, such as age, sex, lifestyle, drugs, supplements, concomitant diseases, and gut microbiome configuration.

\section{Conclusion}

A ketogenic diet is a multi-target nutritional intervention, with sufficient preclinical evidence supporting its beneficial systemic impact, including neurotrophic, antioxidant, neuroprotective, and anti-inflammatory effects. While the ketogenic diet intervention opens new avenues for studying dietary interventions in mood disorders, standardized protocols have to be established. Excellent efficacy and safety data from clinical studies in epilepsy provide a strong rationale for conducting randomized controlled trials involving the use of the ketogenic diet in individuals with MDD.

\section{Author Contributions}

D.S. carried out the literature research, wrote the article, and generated figures. T.L. conceptualized and supervised the manuscript, performed literature research, wrote the manuscript, and illustrated the figure. 


\section{Competing Interests}

The authors have no conflicts of interest pertaining to this article and the research presented herein.

\section{References}

1. Roberts N, Mountjoy-Venning WC, Anjomshoa M, Banoub JA, Yasin YJ. GBD 2017 disease and injury incidence and prevalence collaborators. Global, regional, and national incidence, prevalence, and years lived with disability for 354 diseases and injuries for 195 countries and territories, 1990-2017: A systematic analysis for the Global Burden of Disease Study 2017. Lancet. 2018; 392: 1789.

2. Whooley MA, Wong JM. Depression and cardiovascular disorders. Annu Rev Clin Psychol. 2013; 9: 327-354.

3. Thase ME, Friedman ES, Biggs MM, Wisniewski SR, Trivedi MH, Luther JF, et al. Cognitive therapy versus medication in augmentation and switch strategies as second-step treatments: A STAR*D report. Am J Psychiatry. 2007; 164: 739-752.

4. Rush AJ, Trivedi MH, Wisniewski SR, Nierenberg AA, Stewart JW, Warden D, et al. Acute and longer-term outcomes in depressed outpatients requiring one or several treatment steps: a STAR*D report. Am J Psychiatry. 2006; 163: 1905-1917.

5. Lam RW, Michalak EE, Bond DJ, Tam EM, Axler A, Yatham LN. Which depressive symptoms and medication side effects are perceived by patients as interfering most with occupational functioning? Depress Res Treat. 2012; 2012: 630206.

6. Young SN. Clinical nutrition: 3. The fuzzy boundary between nutrition and psychopharmacology. CMAJ. 2002; 166: 205-209.

7. Elizabeth L, Machado P, Zinöcker M, Baker $P$, Lawrence $M$. Ultra-processed foods and health outcomes: A narrative review. Nutrients. 2020; 12: 1955.

8. Lakhan SE, Vieira KF. Nutritional therapies for mental disorders. Nutr J. 2008; 7: 1-8.

9. Grosso G, Pajak A, Marventano S, Castellano S, Galvano F, Bucolo C, et al. Role of omega-3 fatty acids in the treatment of depressive disorders: a comprehensive meta-analysis of randomized clinical trials. PloS One. 2014; 9: e96905.

10. Petridou ET, Kousoulis AA, Michelakos T, Papathoma P, Dessypris N, Papadopoulos FC, et al. Folate and B12 serum levels in association with depression in the aged: A systematic review and meta-analysis. Aging Ment Health. 2016; 20: 965-973.

11. Bender A, Hagan KE, Kingston N. The association of folate and depression: A meta-analysis. J Psychiatr Res. 2017; 95: 9-18.

12. Tarleton EK, Littenberg B, MacLean CD, Kennedy AG, Daley C. Role of magnesium supplementation in the treatment of depression: A randomized clinical trial. PloS One. 2017; 12: e0180067.

13. Li Z, Li B, Song X, Zhang D. Dietary zinc and iron intake and risk of depression: A meta-analysis. Psychiatry Res. 2017; 251: 41-47.

14. Tian $Y$, Zheng Z, Ma C. The effectiveness of iron supplementation for postpartum depression: $A$ protocol for systematic review and meta-analysis. Medicine. 2020; 99: e23603.

15. Shaw K, Turner J, Mar C. Are tryptophan and 5-hydroxytryptophan effective treatments for depression? A meta-analysis. Aust N Z J Psychiatry. 2002; 36: 488-491. 
16. Wheless JW. History of the ketogenic diet. Epilepsia. 2008; 49: 3-5.

17. Carroll J, Koenigsberger D. The ketogenic diet: A practical guide for caregivers. J Am Diet Assoc. 1998; 98: 316-321.

18. Paoli A, Bosco G, Camporesi EM, Mangar D. Ketosis, ketogenic diet and food intake control: A complex relationship. Front Psychol. 2015; 6: 27.

19. Sukkar SG, Muscaritoli M. A clinical perspective of low carbohydrate ketogenic diets: A narrative review. Front Nutr. 2021; 8: 642628.

20. Fukao T, Lopaschuk GD, Mitchell GA. Pathways and control of ketone body metabolism: On the fringe of lipid biochemistry. Prostaglandins Leukot Essent Fatty Acids. 2004; 70: 243-251.

21. Veech RL. The therapeutic implications of ketone bodies: The effects of ketone bodies in pathological conditions: Ketosis, ketogenic diet, redox states, insulin resistance, and mitochondrial metabolism. Prostaglandins Leukot Essent Fatty Acids. 2004; 70: 309-319.

22. Norwitz NG, Dalai SS, Palmer CM. Ketogenic diet as a metabolic treatment for mental illness. Curr Opin Endocrinol Diabetes Obes. 2020; 27: 269-274.

23. Wells G, Shea B, O'Connell D, Robertson J, Peterson J, Welch V, et al. The Newcastle-Ottawa Scale (NOS) for assessing the quality of nonrandomised studies in meta-analyses [Internet]. Ottawa: Ottawa Hospital Research Institute; 2021. Available from: http://www.ohri.ca/programs/clinical epidemiology/oxford.asp.

24. Kilkenny C, Browne W, Cuthill IC, Emerson M, Altman DG. Animal research: Reporting in vivo experiments: The ARRIVE guidelines. Br J Pharmacol. 2010; 160: 1577-1579.

25. Martinot JL, Hardy P, Feline A, Jean-Damien H, Bernard M, Domini AL, et al. Left prefrontal glucose hypometabolism in the depressed state: A confirmation. Am J psychiatry. 1990; 147: 1313-1317.

26. Dunn RT, Kimbrell TA, Ketter TA, Frye MA, Willis MW, Luckenbaugh DA, et al. Principal components of the beck depression inventory and regional cerebral metabolism in unipolar and bipolar depression. Biol Psychiatry. 2002; 51: 387-399.

27. Fujimoto T, Takeuchi K, Matsumoto T, Fujita S, Honda K, Higashi Y, et al. Metabolic changes in the brain of patients with late-onset major depression. Psychiatry Res Neuroimaging. 2008; 164: 48-57.

28. Kimbrell TA, Ketter TA, George MS, Little JT, Benson BE, Willis MW, et al. Regional cerebral glucose utilization in patients with a range of severities of unipolar depression. Biol Psychiatry. 2002; 51: 237-252.

29. Kahl KG, Georgi K, Bleich S, Muschler M, Hillemacher T, Hilfiker-Kleinert D, et al. Altered DNA methylation of glucose transporter 1 and glucose transporter 4 in patients with major depressive disorder. J Psychiatr Res. 2016; 76: 66-73.

30. Błaszczyk B, Czuczwar SJ. Epilepsy coexisting with depression. Pharmacol Rep. 2016; 68: 10841092.

31. Kugaya A, Sanacora G. Beyond monoamines: Glutamatergic function in mood disorders. CNS Spectr. 2005; 10: 808-819.

32. Brambilla P, Perez J, Barale F, Schettini G, Soares JC. GABAergic dysfunction in mood disorders. Mol Psychiatry. 2003; 8: 721-737.

33. Yudkoff M, Daikhin Y, Nissim I, Horyn O, Lazarow A, Luhovyy B, et al. Response of brain amino acid metabolism to ketosis. Neurochem Int. 2005; 47: 119-128. 
34. Yudkoff M, Daikhin Y, Nissim I, Grunstein R, Nissim I, et al. Effects of ketone bodies on astrocyte amino acid metabolism. J Neurochem. 1997; 69: 682-692.

35. Yudkoff M, Daikhin Y, Horyn O, Nissim I, Nissim I. Ketosis and brain handling of glutamate, glutamine, and GABA. Epilepsia. 2008; 49: 73-75.

36. Betteridge DJ. What is oxidative stress? Metabolism. 2000; 49: 3-8.

37. Tobe EH. Mitochondrial dysfunction, oxidative stress, and major depressive disorder. Neuropsychiatr Dis Treat. 2013; 9: 567-573.

38. Federico A, Cardaioli E, Pozzo P, Formichi P, Gallus GN, Radi E. Mitochondria, oxidative stress and neurodegeneration. J Neurol Sci. 2012; 322: 254-262.

39. Milder J, Patel M. Modulation of oxidative stress and mitochondrial function by the ketogenic diet. Epilepsy Res. 2012; 100: 295-303.

40. Patel M. Mitochondrial dysfunction and oxidative stress: Cause and consequence of epileptic seizures. Free Radic Biol Med. 2004; 37: 1951-1962.

41. Dantzer R. Depression and Inflammation: An intricate relationship. Biol Psychiatry. 2012; 71: 45.

42. Miller $\mathrm{AH}$, Raison $\mathrm{CL}$. The role of inflammation in depression: From evolutionary imperative to modern treatment target. Nat Rev Immunol. 2016; 16: 22-34.

43. Zou W, Feng R, Yang Y. Changes in the serum levels of inflammatory cytokines in antidepressant drug-naïve patients with major depression. PloS One. 2018; 13: e0197267.

44. Dupuis N, Curatolo N, Benoist JF, Auvin S. Ketogenic diet exhibits anti-inflammatory properties. Epilepsia. 2015; 56: e95-e98.

45. Nandivada P, Fell GL, Pan AH, Nose V, Ling PR, Bistrian BR, et al. Eucaloric ketogenic diet reduces hypoglycemia and inflammation in mice with endotoxemia. Lipids. 2016; 51: 703-714.

46. Yang X, Cheng B. Neuroprotective and anti-inflammatory activities of ketogenic diet on MPTPinduced neurotoxicity. J Mol Neurosci. 2010; 42: 145-153.

47. Huang $C$, Wang $P, X u X$, Zhang $Y$, Gong $Y, H u ~ W$, et al. The ketone body metabolite $\beta$ hydroxybutyrate induces an antidepression-associated ramification of microglia via HDACs inhibition-triggered Akt-small RhoGTPase activation. Glia. 2018; 66: 256-278.

48. Yamanashi T, Iwata M, Kamiya N, Tsunetomi K, Kajitani N, Wada N, et al. Beta-hydroxybutyrate, an endogenic NLRP3 inflammasome inhibitor, attenuates stress-induced behavioral and inflammatory responses. Sci Rep. 2017; 7: 1-11.

49. Yirmiya R, Rimmerman N, Reshef R. Depression as a microglial disease. Trends Neurosci. 2015; 38: 637-658.

50. Liwinski T, Leshem A, Elinav E. Breakthroughs and bottlenecks in microbiome research. Trends Mol Med. 2021; 27: 298-301.

51. Zhu S, Jiang $\mathrm{Y}, \mathrm{Xu} \mathrm{K}, \mathrm{Cui} \mathrm{M}, \mathrm{Ye} \mathrm{W}$, Zhao G, et al. The progress of gut microbiome research related to brain disorders. J Neuroinflammation. 2020; 17: 1-20.

52. Zheng $P$, Zeng B, Zhou C, Liu M, Fang Z, Xu X, et al. Gut microbiome remodeling induces depressive-like behaviors through a pathway mediated by the host's metabolism. Mol Psychiatry. 2016; 21: 786-796.

53. Winter G, Hart RA, Charlesworth RP, Sharpley CF. Gut microbiome and depression: What we know and what we need to know. Rev Neurosci. 2018; 29: 629-643. 
54. Valles-Colomer M, Falony G, Darzi Y, Tigchelaar EF, Wang J, Tito RY, et al. The neuroactive potential of the human gut microbiota in quality of life and depression. Nat Microbiol. 2019; 4: 623-632.

55. David LA, Maurice CF, Carmody RN, Gootenberg DB, Button JE, Wolfe BE, et al. Diet rapidly and reproducibly alters the human gut microbiome. Nature. 2014; 505: 559-563.

56. Zhang Y, Zhou S, Zhou Y, Yu L, Zhang L, Wang Y, et al. Altered gut microbiome composition in children with refractory epilepsy after ketogenic diet. Epilepsy Res. 2018; 145: 163-168.

57. Xie G, Zhou Q, Qiu CZ, Dai WK, Wang HP, Li YH, et al. Ketogenic diet poses a significant effect on imbalanced gut microbiota in infants with refractory epilepsy. World J Gastroenterol. 2017; 23: 6164-6171.

58. Nagpal R, Neth BJ, Wang S, Craft S, Yadav H. Modified Mediterranean-ketogenic diet modulates gut microbiome and short-chain fatty acids in association with Alzheimer's disease markers in subjects with mild cognitive impairment. EBioMedicine. 2019; 47: 529-542.

59. Ang QY, Alexander M, Newman JC, Tian Y, Cai J, Upadhyay V, et al. Ketogenic diets alter the gut microbiome resulting in decreased intestinal th17 cells. Cell. 2020; 181: 1263-1275.

60. Swidsinski A, Dörffel Y, Loening-Baucke V, Gille C, Göktas Ö, Reißhauer A, et al. Reduced mass and diversity of the colonic microbiome in patients with multiple sclerosis and their improvement with ketogenic diet. Front Microbiol. 2017; 8: 1141.

61. Olson CA, Vuong HE, Yano JM, Liang QY, Nusbaum DJ, Hsiao EY. The gut microbiota mediates the anti-seizure effects of the ketogenic diet. Cell. 2018; 173: 1728-1741.

62. Cabrera-Mulero A, Tinahones A, Bandera B, Moreno-Indias I, González MM, Tinahones FJ. Keto microbiota: A powerful contributor to host disease recovery. Rev Endocr Metab Disord. 2019; 20: 415-425.

63. Frazer A, Morilak DA. What should animal models of depression model? Neurosci Biobehav Rev. 2005; 29: 515-523.

64. Harro J. Animal models of depression: Pros and cons. Cell Tissue Res. 2019; 377: 5-20.

65. Murphy P, Likhodii S, Nylen K, Burnham WM. The antidepressant properties of the ketogenic diet. Biol Psychiatry. 2004; 56: 981-983.

66. Ari C, Kovács Z, Juhasz G, Murdun C, Goldhagen CR, Koutnik AP, et al. Exogenous ketone supplements reduce anxiety-related behavior in Sprague-Dawley and Wistar Albino Glaxo/Rijswijk rats. Front Mol Neurosci. 2016; 9: 137.

67. Kovács Z, D’Agostino DP, Ari C. Anxiolytic effect of exogenous ketone supplementation is abolished by adenosine $A 1$ receptor inhibition in Wistar Albino Glaxo/Rijswijk rats. Front Behav Neurosci. 2018; 12: 29.

68. Sussman D, Germann J, Henkelman M. Gestational ketogenic diet programs brain structure and susceptibility to depression \& anxiety in the adult mouse offspring. Brain Behav. 2015; 5: e00300.

69. Benjamin JS, Pilarowski GO, Carosso GA, Zhang L, Huso DL, Goff LA, et al. A ketogenic diet rescues hippocampal memory defects in a mouse model of Kabuki syndrome. PNAS. 2017; 114: 125-130.

70. Gumus H, Ilgin R, Koc B, Yuksel O, Kizildag S, Guvendi G, et al. A combination of ketogenic diet and voluntary exercise ameliorates anxiety and depression-like behaviors in Balb/c mice. Neurosci Lett. 2022; 770: 136443. 
71. Kossoff EH, Zupec-Kania BA, Auvin S, Ballaban-Gil KR, Bergqvist AG, Blackford R, et al. Optimal clinical management of children receiving dietary therapies for epilepsy: Updated recommendations of the international ketogenic diet study group. Epilepsia Open. 2018; 3: 175-192.

72. El-Mallakh RS, Paskitti ME. The ketogenic diet may have mood-stabilizing properties. Med Hypotheses. 2001; 57: 724-726.

73. IJff DM, Postulart D, Lambrechts DA, Majoie MH, Kinderen RJ, Hendriksen JG, et al. Cognitive and behavioral impact of the ketogenic diet in children and adolescents with refractory epilepsy: A randomized controlled trial. Epilepsy Behav. 2016; 60: 153-157.

74. Phelps JR, Siemers SV, El-Mallakh RS. The ketogenic diet for type II bipolar disorder. Neurocase. 2013; 19: 423-426.

75. McClernon FJ, Yancy WS, Eberstein JA, Atkins RC, Westman EC. The effects of a lowcarbohydrate ketogenic diet and a low-fat diet on mood, hunger, and other self-reported symptoms. Obesity. 2007; 15: 182.

76. lacovides S, Goble D, Paterson B, Meiring RM. Three consecutive weeks of nutritional ketosis has no effect on cognitive function, sleep, and mood compared with a high-carbohydrate, lowfat diet in healthy individuals: A randomized, crossover, controlled trial. Am J Clin Nutr. 2019; 110: 349-357.

77. Shegelman A, Carson KA, McDonald TJ, Henry-Barron BJ, Diaz-Arias LA, Cervenka MC. The psychiatric effects of ketogenic diet therapy on adults with chronic epilepsy. Epilepsy Behav. 2021; 117: 107807.

78. Rawat $K$, Singh N, Kumari $P$, Saha L. A review on preventive role of ketogenic diet (KD) in CNS disorders from the gut microbiota perspective. Rev Neurosci. 2021; 32: 143-157.

79. Taylor MK, Sullivan DK, Mahnken JD, Burns JD, Swerdlow RH. Feasibility and efficacy data from a ketogenic diet intervention in Alzheimer's disease. Alzheimers Dement. 2018; 4: 28-36.

80. Arya R, Peariso K, Gaínza-Lein M, Harvey J, Bergin A, Brenton JN, et al. Efficacy and safety of ketogenic diet for treatment of pediatric convulsive refractory status epilepticus. Epilepsy Res. 2018; 144: 1-6.

81. Bostock EC, Kirkby KC, Taylor BV. The current status of the ketogenic diet in psychiatry. Front Psychiatry. 2017; 8: 43.

82. Yaroslavsky Y, Stahl Z, Belmaker RH. Ketogenic diet in bipolar illness. Bipolar Disord. 2002; 4: 75. 


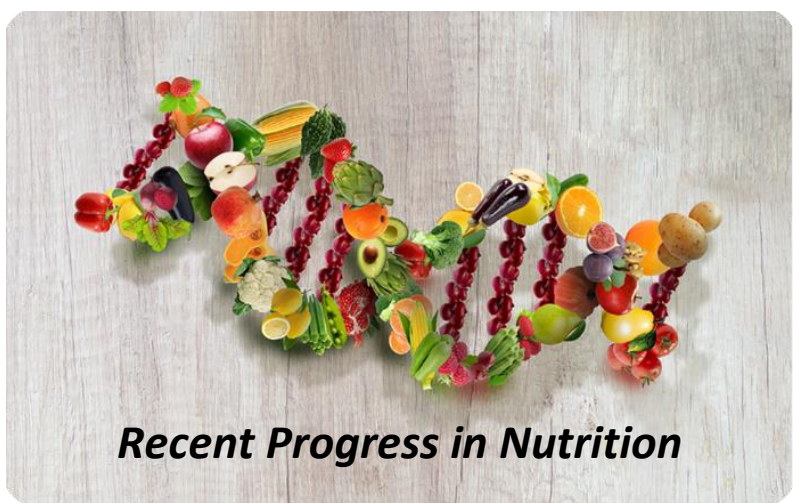

Enjoy Recent Progress in Nutrition by:

1. Submitting a manuscript

2. Joining in volunteer reviewer bank

3. Joining Editorial Board

4. Guest editing a special issue

For more details, please visit:

http://www.lidsen.com/journals/rpn 